\title{
Distance scale calibration based on early-type binaries
}

\author{
Mónica Taormina ${ }^{1, \star}$, Grzegorz Pietrzyński ${ }^{1}$, Bogumił Pilecki ${ }^{1}$, lan Thompson², and Dariusz \\ Graczyk $^{1}$ \\ ${ }^{1}$ Centrum Astronomiczne im. Mikołaja Kopernika (CAMK), PAN, Bartycka 18, 00-716 Warsaw, Poland \\ ${ }^{2}$ Carnegie Observatories, 813 Santa Barbara Street, Pasadena, CA 91101-1292, USA
}

\begin{abstract}
Our main goal is to establish a firm empirical calibration of the surface brightness vs. $(V-K)$ color relation for early type stars based on high quality spectroscopic and infrared observations of early-type detached eclipsing systems in the Large Magellanic Cloud (LMC). Our calibration of this relation will allow distance determinations accurate to about $2.5 \%$ to a single object located well beyond the Magellanic Clouds. This will let us calibrate other distance indicators, including period-luminosity relations for Cepheids. The first step of the project is to determine precise parameters for a sample of B-type systems in the LMC. We have already made a preliminary analysis of light and radial velocity curves for selected objects and measured their masses and radii. Here we present the results for one such system.
\end{abstract}

\section{Introduction}

Detached eclipsing double-lined spectroscopic binaries (SB2) offer a unique opportunity to measure directly, and accurately, stellar parameters like mass, luminosity, and radius. For such systems linear dimensions can be measured with a precision of $1 \%$ from the analysis of light and radial velocity curves. The distance to an eclipsing binary follows from the linear radii and the angular diameters derived from the absolute surface brightness coming from the surface brightness (SB) vs. $(V-K)$ color calibration. The latter is already well established for late type stars for which accurate stellar angular diameters were determined by interferometry ([2]).

Not much work, however, has been done so far for the early type stars, as there is a lack of such objects in the Solar neighborhood, for which parallaxes or interferometric data could be available (we need independent distance or angular diameter determination). In the latest work on that subject Challouf et al. ([1]) have observed interferometrically a sample of such stars, but could only reduce the uncertainty from about $10 \%$ to $7 \%$, which is not enough for precise distance measurements.

Our idea is to observe early-type systems in the Large Magellanic Cloud (LMC) for which we know the distance with a very good accuracy. Using this distance together with the radii from the modeling we can calculate the angular diameters of the stars. With these diameters and observed magnitudes we will be able to calculate the SB and determine the SB-color relation (SBCR) for early type stars with unprecedented precision.

When applied for the early-type stars the eclipsing binary method will serve to accurately measure distances to the galaxies in the Local Group and beyond. Having reliable distances to numerous galaxies with different environmental conditions will be of high importance for the calibration of other standard candles and especially period-luminosity relations for Classical Cepheids. This in turn is one of the most important steps to calibrate the cosmic distance scale.

\section{Data}

We have selected a sample of 9 objects from the catalog of about 30000 eclipsing binaries in the LMC recently produced by the OGLE collaboration ([3]). The sample consists of detached systems with

^taormina@camk.edu.pl 

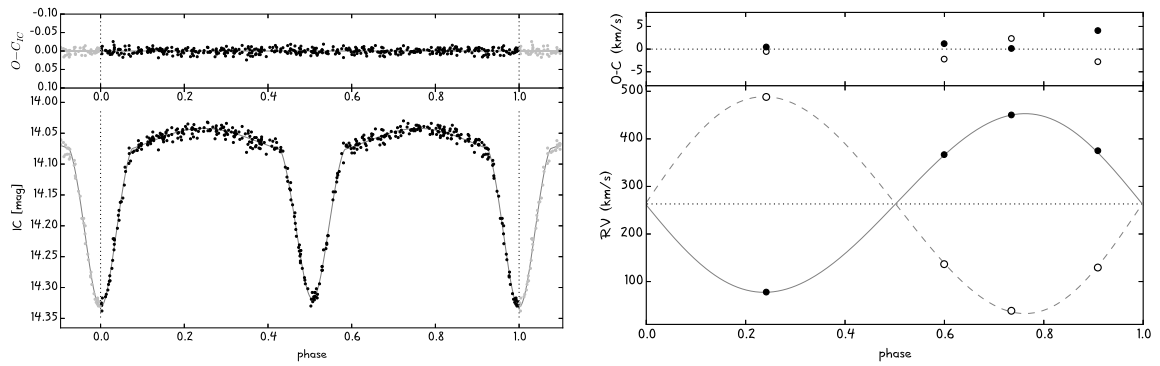

Figure 1. Light and radial velocity curve for one of the system with corresponding preliminary models.

well-covered deep eclipses of similar depths. They are the best-ever discovered objects for the precise parameter determination, which is necessary to calibrate the SBCR for early-type stars.

For the calibration of the SBCR apart from the optical photometry we will need good-quality radial velocity curves and infrared photometry. In the preliminary study we have already collected at least three spectra for all the stars with the MIKE and UVES instruments mounted on the 6.5-meter Magellan Telescope and 8.2-meter VLT, respectively.

\section{Analysis and first results}

We have performed a simple modeling of all the systems from the sample of 9 eclipsing binaries using both JKTEBOP code and Wilson-Devinney code with the PHOEBE GUI. Using these preliminary models we were able to look for the stars for which the most important parameters can be determined with the lowest possible uncertainty, in particular we made sure that the radii can be measured with a precision better than $1 \%$.

For one object we have obtained 4 observations covering both quadratures and thus letting us check the lower limit of the precision we will get for our objects. Our measured velocities have a scatter of about $2 \mathrm{~km} / \mathrm{s}$ while the velocity semi-amplitudes are of the order of $200 \mathrm{~km} / \mathrm{s}$ (see Fig. 1). The radial velocities were measured using the Broadening Function technique ([4]). This technique is superior to the commonly used cross-correlation function as it gives narrower and stronger profiles.

From the preliminary model the masses of the components are 15 and $12 M_{\odot}$, and their radii 8 and $7 R_{\odot}$, with the separation between the stars of $30 R_{\odot}$. The system is a well detached one and will serve for the calibration of the SBCR.

Acknowledgments: Support from the IdP II 2015000264 grant of the Polish Ministry of Science and Higher Education.

\section{References}

[1] Challouf, M., Nardetto, N., Mourard, D., et al., A\&A, 570, A104 (2014)

[2] di Benedetto, G. P., MNRAS, 357, 174 (2005)

[3] Graczyk, D., Soszyński, I., Poleski, R., et al., Acta Astron., 61, 103 (2011)

[4] Rucinski, S., in Precise Stellar Radial Velocities, IAU Colloq. 170, ed. J. B. Hearnshaw \& C. D. Scarfe, Astronomical Society of the Pacific Conference Series, 185, 82 (1999) 